\title{
Wearable E-Textile Wireless RF Power Supply based on a Textile Supercapacitor and a Flexible Rectenna Filament
}

\author{
Mahmoud Wagih ${ }^{1}$, Graduate Student Member, IEEE, Nicholas Hillier ${ }^{2}$, Sheng Yong ${ }^{1}$, Alex S. Weddell ${ }^{1}$, \\ Member, IEEE, and Steve Beeby ${ }^{1}$, Senior Member, IEEE \\ ${ }^{1}$ School of Electronics and Computer Science, University of Southampton \\ ${ }^{2}$ Energy Storage CDT, University of Southampton \\ mahm1g15@ecs.soton.ac.uk,nh3g09@soton.ac.uk, S.Yong@soton.ac.uk, asw@ecs.soton.ac.uk, spb@ecs.soton.ac.uk
}

\begin{abstract}
Sustainable battery-free operation is a requirement for wearable electronic textiles. This work presents a complete wearable textile-based radio frequency energy harvester and an energy storage module. The rectifying-antenna (rectenna) receives incident sub-1 GHz $\mathrm{RF}$ power from a license-free transmitter and converts it to DC with up to $90 \%$ efficiency and $8 \mathrm{~V}$ DC output from under $4 \mathrm{~mW}$ of RF power. A spraycoated textile $7.1 \mathrm{mF}$ supercapacitor is integrated as an energy storage unit. Electrochemical Impedance Spectroscopy has been used to characterize the supercapacitor's impedance and a fourbranch circuit model has been obtained. The $7.1 \mathrm{mF}$ textile supercapacitor has been demonstrated charging to $1 \mathrm{~V}(3.55 \mathrm{~mJ})$ in under 11 seconds at $1.2 \mathrm{~m}$ separation from a wireless power source, demonstrating over $15 \%$ end-to-end efficiency, the highest charging efficiency of a textile supercapacitor from an integrated flexible energy source.
\end{abstract}

Index Terms-Antenna, E-textiles, Rectenna, RF Energy Harvesting, Supercapacitor, Wireless Power Transfer

\section{INTRODUCTION}

Integrating electronic functionalities, such as sensing and wireless communication, in garments using textile materials, known as e-textiles, represents an emerging sector of the Internet of Things (IoT) market. Enabling such devices to be power-autonomous and battery-free through sustainable energy harvesting and delivery methods is key to e-textiles becoming ubiquitous in garments.

Textile-based ferroelectric [1], tribolectric [2] and thermoelectric [3] energy harvesting has been previously reported. Furthermore, near-field and far-field wireless power transfer (WPT) has been reported using textile-based coils [4], [5] and flexible antennas integrated in textiles [6] or fabricated using conductive fabrics [7], [8]. As opposed to ambient energy harvesters which depend on the user's activity, onbody placement, or certain operation conditions such as steep thermal gradients [3], far-field WPT using rectifying antennas (rectennas) enables reliable delivery of power with theoretical Radio Frequency (RF) to DC efficiencies approaching $100 \%$.

Recent advances in energy storage on textile materials has shown the potential to store milli-Joules of energy in

This work was supported by the European Commission through the project EnABLES: European Infrastructure Powering the Internet of Things, funded under H2020-EU.1.4.1.2. and by the UK EPSRC through EP/L016818/1
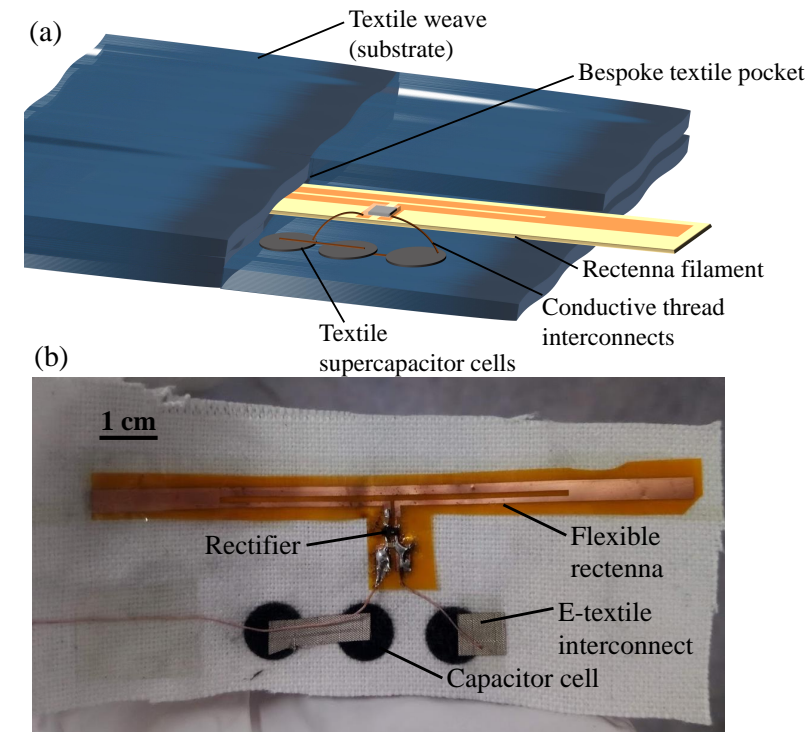

Fig. 1. The proposed textile wireless power supply module: (a) diagram showing the system's main components, (b) photograph of the prototype implemented on a cotton substrate.

textile supercapacitors or batteries [1], [9]. However, when integrating such storage devices with mechanical energy transducers the charging efficiency is adversely affected, due to the impedance-mismatch at the harvester-capacitor interface, and the harvester's low-frequency. Thus, it can take up to one hour to charge a $2 \mathrm{mF}$ flexible supercapacitor to $0.5 \mathrm{~V}$, with an end-to-end efficiency of less than $1 \%$ [1]. Moreover, utilizing RF energy harvesting to charge a sensor node has only been considered using standard rigid rectennas and supercapacitors, with an intermediate power management interface [10].

This work presents a textile-based power supply and energy storage module based on a flexible rectenna integrated with an in-house spray-coated textile supercapacitor. The proposed module, shown in Fig. 1, is able to store up to $8.2 \mathrm{~mJ}$ of energy in under 30 seconds of wireless charging from a 915 $\mathrm{MHz}$ transmitter at $1.2 \mathrm{~m}$ from the harvester, demonstrating over $15 \%$ end-to-end charging efficiency. This paper discusses 
the implementation of the textile-integrated rectenna (section II), the textile supercapacitor characterization (section III) and evaluates the performance of the textile power supply module under different RF power excitations (section IV).

\section{Energy Generation: The Rectenna}

To harvest or receive far-field wireless power, the receiving antenna needs to be matched to the rectifier's impedance. This can be achieved using either a stand-alone impedance transformer (a matching network) or an antenna designed with a complex impedance which directly conjugates the rectifier's impedance [11]. The key figure-of-merit when designing a rectenna is the Power Conversion Efficiency (PCE), given by the ratio of the harvested DC power across a resistive $\left(Z_{\text {load }}\right)$ load to the available RF power at the antenna's port $P_{R F}(1)$.

$$
P C E=\frac{V_{\text {load }}^{2}}{Z_{\text {load }} P_{R F}}
$$

The maximum achievable PCE is a function of the rectifying diode (due to the forward voltage drop across the junction), and the impedance matching quality between the antenna and the rectifier. In this work, antenna-rectifier co-design is chosen to eliminate the separate impedance-matching stage to minimize the system's form-factor. In addition, it has been shown that a rectenna without a matching network can be designed to achieve the highest PCE compared to rectennas with a matching network based on an iterative optimization process [12].

The diode selected in this work is the Infineon BAT15-04R, due to its relatively high break-down voltage of $4 \mathrm{~V}$ and low forward voltage drop of $230 \mathrm{mV}$, making it suitable for a wide dynamic range of RF power levels which enables rapid and efficient charging of the textile energy storage. The optimal input impedance of the rectenna, as well as the rectifier's performance, have been simulated using non-linear harmonic balance simulation in Keysight Advanced Design Systems (ADS). Fig. 2 shows the simulated PCE and DC voltage across a $20 \mathrm{k} \Omega$ load. A folded dipole antenna enables achieving a tunable high-impedance that can directly match the rectifier. The antenna has been tuned based on the parametric analysis in [12] to match the rectifier's impedance at $0 \mathrm{dBm}$.

The rectenna has been fabricated on a flexible polyimide filament of less than $50 \mu \mathrm{m}$ thickness. The filament can then be integrated into a standard textile weave as well as encapsulated using vacuum forming or glob-top epoxy encapsulation. The antenna's traces have been etched using standard photolithography, this process has been previously utilized to realize RF harvesters [13] and textile antennas up to millimetre-wave bands [14]. Fig. 3 shows a photograph of the rectenna inserted in a bespoke woven textile pocket.

\section{Textile Supercapacitor Characterization AND MODELLING}

Electrochemical Impedance Spectroscopy (EIS) has been used to characterize the impedance of the in-house textile supercapacitor over frequencies ranging from 0.1 to $100 \mathrm{~Hz}$

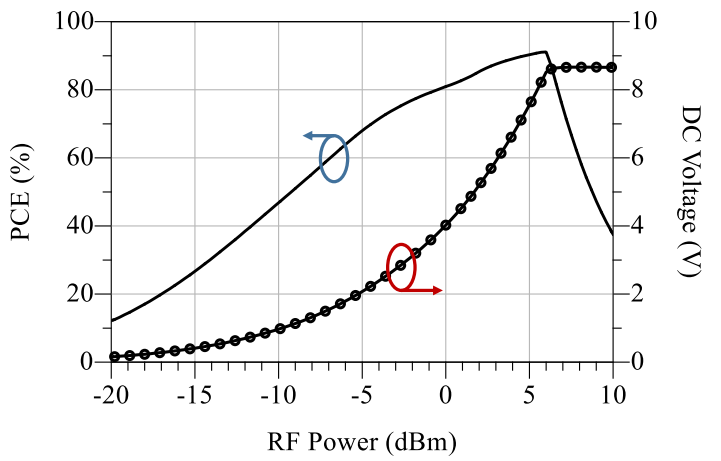

Fig. 2. PCE and DC voltage output of the flexible rectenna across a $20 \mathrm{k} \Omega$ resistive load at $915 \mathrm{MHz}$.

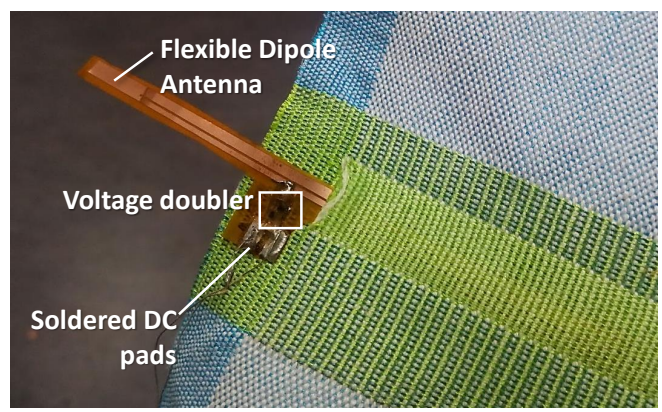

Fig. 3. Photograph of the flexible folded-dipole rectenna integrated in the bespoke textile pocket.

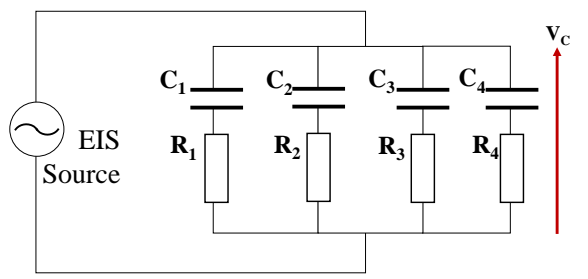

Fig. 4. Empirical circuit model of the textile supercapacitor. $C_{1}=7.1 \mathrm{mF}$, $C_{2}=2.6 \mathrm{mF}, C_{3}=0.7 \mathrm{mF}, C_{4}=47 \mu \mathrm{F}, R_{1}=488 \Omega, R_{2}=117 \Omega, R_{3}=66.9 \Omega$, $R_{4}=107 \Omega$.

[1]. Three individual capacitor cells, each rated for $1.2 \mathrm{~V}$, were connected in series to allow the capacitor's maximum rated voltage to $3.6 \mathrm{~V}$ to match the rectenna's output. A four branch supercapacitor model has been used to model the textile supercapacitor [15]. Unlike the complete model in [15], the voltage-dependent capacitance has not been considered due to its minimal influence on the supercapacitor's overall performance. The empirical circuit model, shown in Fig. 4, matches the measured input impedance up to $100 \mathrm{~Hz}$ with less than $8 \%$ error in $C_{1}$, and less than 5\% error for the other capacitive branches compared to the EIS measurements.

\section{Integrated Wireless Power Supply Module EVALUATION}

\section{A. Capacitor Charging using an RF Signal Generator}

A power-calibrated continuous wave RF signal generator has been used initially to feed the rectifier. This enables 


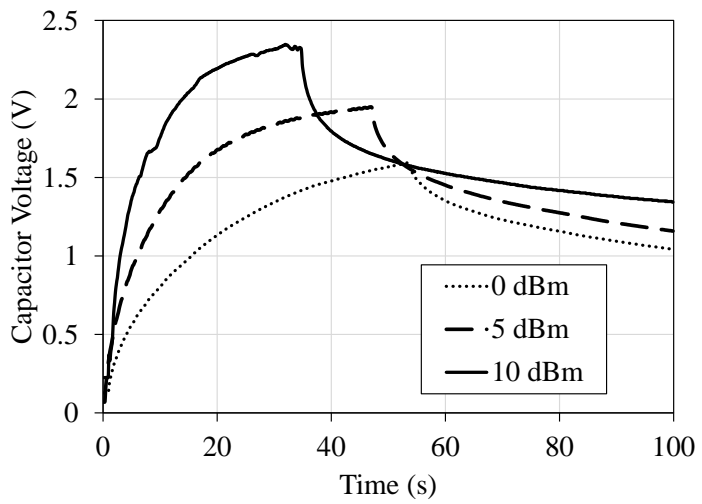

Fig. 5. Charging voltage of the textile supercapacitor using different power levels of RF power.

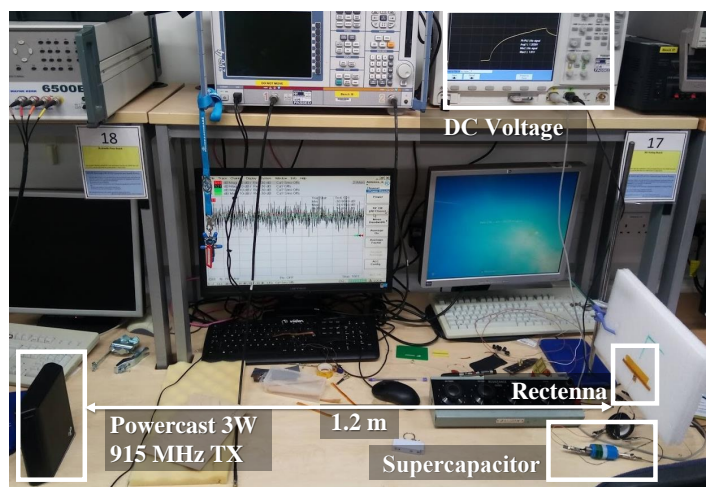

Fig. 6. Test setup showing the wireless powering of the textile supercapacitor using the rectenna filament at $1.2 \mathrm{~m}$ from the Powercast transmitter.

characterizing the rectifier's DC output with $0.1 \mathrm{~dB} P_{R F}$ accuracy compared to wireless testing. A $2 \mathrm{k} \Omega$ load has been used to measure the DC power generated by the rectifier. Three RF power levels were considered 0,5 , and $10 \mathrm{dBm}$, the corresponding DC output of the rectifier is $0.41,1.05$, and $2.22 \mathrm{~mW}$, respectively. Fig. 5 shows the charging curves of the supercapacitor under the three different excitations.

It is observed that from less than $3.2 \mathrm{~mW}(5 \mathrm{dBm})$ of $\mathrm{RF}$ power, the proposed RF power supply module is able to store $11.4 \mathrm{~mJ}$ of energy in the textile capacitor in 30 seconds of RF-powering.

\section{B. Wireless Testing using a License-Free Transmitter}

A commercial wireless power transmitter (Powercast) operating at the $915 \mathrm{MHz}$ license-free band has been used to test the rectenna filament integrated with the textile supercapacitor. The Powercast transmitting $3 \mathrm{~W}$ equivalent isotropic radiated power (EIRP) $P_{R F}$ has been placed at $1.2 \mathrm{~m}$ from the rectenna. The rectenna's output has been connected to the supercapacitor using silk-coated fabric Litz wires. The supercapacitor has been concealed in a Swagelok case to prolong its lifetime and improve the repeatability of the experiments. Fig. 6 shows the test setup of the rectenna charging the textile supercapacitor.

The RF power received by the rectenna can be calculated using the Friis free space propagation model using $1.8 \mathrm{dBi}$

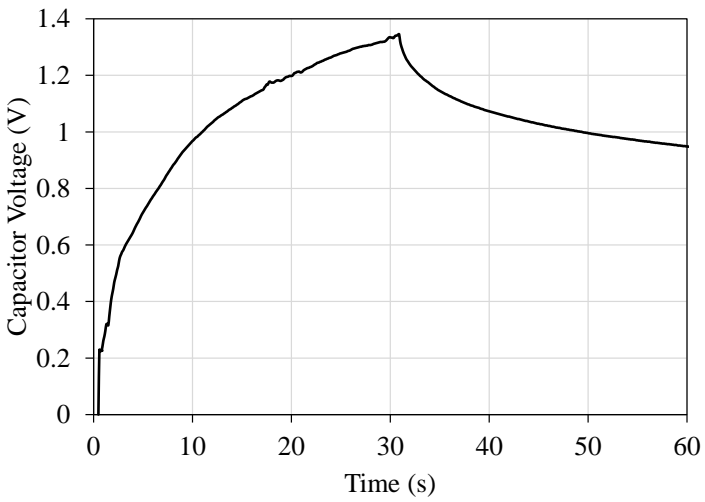

Fig. 7. Wireless charging of the textile supercapacitor at $1.2 \mathrm{~m}$ separation from the Powercast transmitter.

receiver gain, 3W EIRP at $915 \mathrm{MHz}$ and $1.2 \mathrm{~m}$ separation. The calculated received power using the setup in Fig. 6 is $3.32 \mathrm{dBm}(2.14 \mathrm{~mW})$. However, due to multi-path effects and impedance mismatch between the antenna and the rectifier, the effective power delivered to the rectifier will be lower. The wireless power transmitter is turned on for 30 seconds before the capacitor is allowed to self-discharge. Fig. 7 shows the supercapacitor's charging curve using the wireless power source at $1.2 \mathrm{~m}$ separation. In 11 seconds, the capacitor stores $3.55 \mathrm{~mJ}$ of energy. The average charging power $P_{D C}$ of the capacitor $(C=7.1 \mathrm{mF})$ can be used to calculate the average end-to-end efficiency (2), where $t$ is the $11 \mathrm{~s}$ charging time, $V_{C}$ is the voltage across the capacitor, and $P_{R F}$ is the 2.14 $\mathrm{mW}$ RF power level.

$$
\eta_{\text {charging }}=\frac{P_{D C}}{P_{R F}}=\frac{C V_{c}^{2}}{2} \times \frac{1}{t} \times \frac{1}{P_{R F}}
$$

The calculated net average DC power yield is $322 \mu \mathrm{W}$ demonstrating a $15 \%$ end-to-end efficiency. This is inclusive of the antenna and rectifier's losses, the impedance mismatch between the antenna and the capacitor, as well as the selfdischarge in the textile supercapacitor. This represents the highest reported charging efficiency of textile supercapacitor using an integrated harvester.

\section{CONCLUSION}

This work has presented a RF wireless power supply with integrated energy storage for e-textile applications, based on a flexible rectenna filament and a spray-coated textile supercapacitor. The proposed unit can successfully store more than $3.55 \mathrm{~mJ}$ of energy in under 11 seconds of charging using a commercial wireless power source at $1.2 \mathrm{~m}$ separation, with an end-to-end efficiency of $15 \%$, the highest charging efficiency of a textile supercapacitor using a wearable energy source. This demonstrates the feasibility of using RF power to entirely charge a textile-based energy storage device enabling batteryfree wearable sensing and wireless communication. 


\section{REFERENCES}

[1] S. Yong, J. Shi, and S. Beeby, "Wearable Textile Power Module Based on Flexible Ferroelectret and Supercapacitor," Energy Technology, vol. 7 (5), pp. $2284-2291,2018$.

[2] W. Paosangthong, M. Wagih, R. Torah, and S. Beeby, "Textile-based triboelectric nanogenerator with alternating positive and negative freestanding grating structure," Nano Energy, vol. 66, 2019.

[3] V. Leonov, "Thermoelectric Energy Harvesting of Human Body Heat for Wearable Sensors," IEEE Sensors J., vol. 13 (6), pp. 2284 - 2291, 2013.

[4] M. Wagih, A. Komolafe, and B. Zaghari, "Dual-Receiver Wearable 6.78 MHz Resonant Inductive Wireless Power Transfer Glove Using Embroidered Textile Coils," IEEE Access, vol. 8, pp. 24630 - 24642, 2020.

[5] S. H. Kang, V. T. Nguyen, and C. W. Jung, "Analysis of MR-WPT using planar textile resonators for wearable applications," IET Microwaves, Antennas \& Propagation, vol. 10, 14, pp. 1541 - 1546, 2016.

[6] M. Wagih, A. S. Weddell, and S. Beeby, "Sub-1 GHz Flexible Concealed Rectenna Yarn for High-Efficiency Wireless-Powered Electronic Textiles," in 2020 European Conference on Antennas and Propagation (EuCAP), 2020.

[7] G. Monti, L. Corchia, and L. Tarricone, "UHF Wearable Rectenna on Textile Materials," IEEE Trans. Antennas. Propag., vol. 61, 7, pp. 3869 $-3873,2013$.

[8] S. Lemey, S. Agneessens, and H. Rogier, "Wearable smart objects: Microwaves propelling smart textiles: A review of holistic designs for wireless textile nodes," IEEE Microwave Magazine, vol. 19, 6, pp. 83100,2018

[9] Y. Li, N. Hillier, S. Yong, S. Arumugam, C. Craig, D. Harrowven, and S. Beeby, "Water Activated Primary Textile Battery," in 2019 IEEE International Conference on Flexible and Printable Sensors and Systems (FLEPS), 2019.

[10] A. Okba, A. Takacs, and H. Aubert, "Compact rectennas for ultra-lowpower wireless transmission applications," IEEE Trans. Microw. Theory Techn., vol. 67, 5, pp. 1697 - 1707, 2019.

[11] M. Wagih, A. S. Weddell, and S. Beeby, "Rectennas for RF Energy Harvesting and Wireless Power Transfer: a Review of Antenna Design," IEEE Antennas and Propagation Magazine, vol. Accepted: In Press, 2019.

[12] M. Wagih, A. S. Weddell, and S. Beeby, "High-Efficiency Sub-1 GHz Flexible Compact Rectenna based on Parametric Antenna-Rectifier CoDesign," in 2020 IEEE MTT-S International Microwave Symposium, 2020.

[13] J. Bito, J. G. Hester, and M. M. Tentzeris, "Ambient rf energy harvesting from a two-way talk radio for flexible wearable wireless sensor devices utilizing inkjet printing technologies," IEEE Trans. Microw. Theory Techn., vol. 63, 12, pp. 4533-4543, 2015.

[14] M. Wagih, A. S. Weddell, and S. Beeby, "Millimeter-Wave Textile Antenna for On-Body RF Energy Harvesting in Future 5G Networks," in 2019 IEEE Wireless Power Transfer Conference (WPTC), 2019.

[15] L. Zubieta and R. Bonert, "Characterization of double-layer capacitors for power electronics applications," IEEE Trans. Ind. Applications, vol. 36 (1), pp. $199-205,2000$. 